
allemande

47-2 | 2015

Retour sur le modèle Rhénan : humanisme, capitalisme et métropolisation | Patrimonialisation du passé « allemand » en Europe centrale après 1990

\title{
Patrimonialisation du passé allemand ? Une exposition sur la Haute-Silésie allemande en Haute- Silésie polonaise
}

\section{Pascal Fagot}

\section{(QpenEdition \\ Journals}

\section{Édition électronique}

URL : https://journals.openedition.org/allemagne/326

DOI : $10.4000 /$ allemagne.326

ISSN : 2605-7913

Éditeur

Société d'études allemandes

\section{Édition imprimée}

Date de publication : 16 décembre 2015

Pagination : 489-500

ISSN : 0035-0974

Référence électronique

Pascal Fagot, «Patrimonialisation du passé allemand? Une exposition sur la Haute-Silésie allemande en Haute-Silésie polonaise ", Revue d'Allemagne et des pays de langue allemande [En ligne], 47-2 | 2015, mis en ligne le 13 décembre 2017, consulté le 18 mai 2021. URL : http://journals.openedition.org/ allemagne/326 ; DOI : https://doi.org/10.4000/allemagne.326 


\section{Patrimonialisation du passé allemand? Une exposition sur la Haute-Silésie allemande en Haute-Silésie polonaise}

- Pascal Fagot*

\section{Introduction}

Le thème de ces journées d'étude «Patrimonialisation du passé allemand en Europe centrale. Enjeux de représentation » pose tout d'abord la question de la définition des concepts de patrimoine, et notamment de patrimoine culturel, et de patrimonialisation. Pour commencer, nous avons tout simplement consulté Wikipedia qui, pour la partie française de l'encyclopédie, propose la définition suivante du patrimoine culturel: «L'ensemble des biens, matériels ou immatériels, ayant une importance artistique et/ou historique certaine, et qui appartiennent soit à une entité privée (personne, entreprise, association, etc.), soit à une entité publique (commune, département, région, pays, etc.) [...] Le patrimoine fait appel à l'idée d'un héritage légué par les générations qui nous ont précédés, et que nous devons transmettre intact ou augmenté aux générations futures, ainsi qu'à la nécessité de constituer un patrimoine pour demain ${ }^{(1)}$.

En allemand, le terme de Kulturerbe mène à celui de Kulturgut, dont Wikipedia offre une définition très évasive qu'elle emprunte à Duden : "Ce qui existe en tant que valeur culturelle et qui est conservé " ${ }^{(2)}$. Duden Online complète cette définition de la façon suivante: «Bien culturel dont a hérité une communauté, un peuple ${ }^{(3)}$.

L'entrée polonaise, dont la traduction serait " héritage culturel », définit le patrimoine comme « un ensemble de choses immobilières et mobilières avec les valeurs spirituelles, les phénomènes historiques et sociaux qui y sont liés, reconnues comme dignes d'une protection juridique pour le bien de la société et son développement et comme dignes d'être transmises aux générations prochaines eu égard aux valeurs

* Professeur des universités, Université de Strasbourg, EA 1341 «Études germaniques ».

1 https://fr.wikipedia.org/wiki/Patrimoine_culturel, les sites Internet ont été consultés le 30.03.2015.

2 Ibid.: «Etwas, was als kultureller Wert Bestand hat und bewahrt wird ».

3 http://www.duden.de/rechtschreibung/Kulturerbe : "Überliefertes Kulturgut einer Gemeinschaft, eines Volkes ». 
historiques, patriotiques, religieuses, scientifiques et artistiques généralement comprises et admises $"{ }^{(4)}$.

Ces trois définitions proposées par Wikipedia suggèrent la multiplicité des définitions et surtout des possibilités de mise en pratique de la notion de patrimoine, propre à chacun des groupes mentionnés. Peu satisfait de ce que proposait Wikipedia, nous nous sommes mis à la recherche d'un dénominateur commun que nous avons trouvé dans la définition du patrimoine culturel par le Conseil de l'Europe:

«a) Le patrimoine culturel constitue un ensemble de ressources héritées du passé que des personnes considèrent, par-delà le régime de propriété des biens, comme un reflet et une expression de leurs valeurs, croyances, savoirs et traditions en continuelle évolution. [...]

b) Une communauté patrimoniale se compose de personnes qui attachent de la valeur à des aspects spécifiques du patrimoine culturel qu'elles souhaitent, dans le cadre de l'action publique, maintenir et transmettre aux générations futures ${ }^{(5)}$.

On retient de cette dernière définition que le patrimoine n'est pas une donnée historique, mais une construction collective à partir d'interprétations du concept dont Wikipedia révèle la diversité: des personnes formant une communauté (désignée par un «nous » énigmatique dans la définition proposée par Wikipedia en langue française) sélectionnent dans le matériau du passé des « ressources " dont la spécificité réside dans le fait qu'elles peuvent être exploitées comme vecteur d'un héritage qu'une génération désire transmettre aux générations suivantes.

À partir de cette sélection, cette communauté se confectionne un récit au moyen duquel elle esquisse une certaine représentation d'elle-même qu'elle prétend extraire du passé pour la projeter dans l'avenir. Le discours patrimonial qu'elle se fabrique participe donc de sa construction identitaire.

Le problème que posent a priori les concepts de patrimonialisation et de patrimoine, dérivés du latin pater, tient aux critères de sélection des éléments que chaque groupe retient pour qu'ils figurent dans son patrimoine. Car si l'héritage paternel peut être constitué de valeurs jugées positives, il est également possible qu'il comprenne des parties dont le légataire préfèrerait ne pas hériter. En permettant d'exclure du récit de l'histoire les aspects jugés indignes d'être retenus comme portion du patrimoine et non représentatifs des valeurs que la communauté revendique, le processus de patrimonialisation peut donc être suspecté de passer l'histoire à un tamis dont la fonction serait de filtrer parmi l'héritage à transmettre les seuls éléments fondant une écriture de l'histoire corrigée, consensuelle, apaisante et apaisée.

Pour réfléchir au concept de patrimonialisation, nous nous appuierons ici sur une exposition qui proposait une représentation jusqu'alors inédite en Pologne de l'histoire de la Haute-Silésie. Intitulée Germania, cette exposition fut réalisée en 2012 au

4 http://pl.wikipedia.org/wiki/Dziedzictwo_kulturowe: « Dziedzictwo kulturowe - zasób rzeczy nieruchomych i ruchomych wraz ze związanymi z nim wartościami duchowymi, zjawiskami historycznymi i obyczajowymi, uznawany za godny ochrony prawnej dla dobra społeczeństwa i jego rozwoju oraz przekazania następnym pokoleniom z uwagi na zrozumiałe i akceptowane wartości historyczne, patriotyczne, religijne, naukowe i artystyczne [...]».

5 http://conventions.coe.int/treaty/fr/treaties/html/199.htm. 
musée de Gliwice, ville située en Haute-Silésie aujourd'hui polonaise, mais allemande jusqu'en 1945, qui a porté jusqu'à la fin de la Seconde Guerre mondiale le nom de Gleiwitz ${ }^{(6)}$. La première partie sera consacrée au rappel de ce qu'est et a été la HauteSilésie, dont nous constaterons qu'elle est particulièrement intéressante dans le cadre d'une réflexion sur le patrimoine et la patrimonialisation. Dans un second temps, nous exposerons la rupture ou la révolution patrimoniale dont cette région a été l'objet après 1945. Dans la dernière partie, nous présenterons l'exposition Germania réalisée en 2012 par le musée de Gliwice, en nous demandant s'il s'agit là effectivement d'une «patrimonialisation du passé allemand » en Pologne ou si nous avons affaire à un autre phénomène qu'il nous faudra définir.

\section{La Haute-Silésie: retour sur une histoire}

Dans les colloques, le concept de «Silésie » renvoie généralement à la Basse-Silésie et à sa grande et prestigieuse capitale, Breslau jusqu'en 1945, aujourd'hui Wrocław; il est extrêmement rare qu'on évoque la Haute-Silésie aujourd'hui polonaise, région minière et industrielle située aux confins orientaux du II Reich allemand et jusqu'en 1918 au point de rencontre des Empires allemand, autrichien et russe. Battant tous les records de pollution jusqu'à ce que les mines et les aciéries aient été obligées de fermer à partir des années 1990, cette région fut alors brutalement frappée par une crise dont elle peine aujourd'hui à se relever; son paysage rappelle étrangement celui de la Ruhr et, malgré sa richesse architecturale, sa réputation de région industrielle a pour effet qu'il ne viendrait pas à l'esprit des nombreux touristes visitant le camp de concentration d'Auschwitz tout proche d'étendre à la Haute-Silésie leur curiosité touristique.

Ce n'est que depuis 1945 que Gliwice se trouve en Pologne, à plus de $300 \mathrm{~km}$ à l'est de la frontière germano-polonaise. Entre 1742, l'année où elle fut enlevée à l'Autriche par la Prusse, et 1921, la Haute-Silésie - et avec elle Gleiwitz - s'était en effet trouvée tout entière à l'intérieur des frontières de la Prusse puis du Reich allemand. Quand, en 1918, à la suite de la Première Guerre mondiale, il fut décidé que la Pologne serait reconstituée à partir de territoires appartenant à l'Allemagne, à l'Autriche et à la Russie/Union soviétique, et qu'il s'est agi de dessiner les frontières de ce nouvel État, la riche et très industrielle Haute-Silésie a été vivement disputée par l'Allemagne et la Pologne qui prétendaient chacune être en droit d'en réclamer pour elle la population.

En effet, si les Haut-Silésiens étaient jusqu'en 1945 de citoyenneté allemande et mâ̂trisaient généralement la langue allemande que leur avait imposée le système scolaire, ils étaient nombreux, notamment à la campagne, à parler également un dialecte polonais péjorativement appelé «wasserpolnisch», qui était certes fortement influencé par l'allemand, mais qui leur donnait un accès facile à la langue et au sentiment national polonais. On pourrait citer quantité d'œuvres plus ou moins littéraires présentant le bilinguisme de cette région alors frontalière et faisant état d’une non-coïncidence

6 http://www.muzeum.gliwice.pl/germania-niemiecka-politycznosc-na-gornym-slasku-1871-1945/. L'exposition est complétée par un catalogue sur lequel nous nous sommes également appuyé pour la rédaction de ce texte: Stefan Rosenbaum, Germania. Niemiecka polityczność na Górnym Ślasku 1871-1945 (Germania. La vie politique allemande en Haute-Silésie 1871-1945), Gliwice, Muzeum w Gliwicach, 2012. 
entre langue de communication et sentiment d'appartenance à une nation ${ }^{(7)}$. Comme la région était riche du charbon qui se trouvait dans son sol et de l'industrie lourde qui s'y était développée depuis le XIX ${ }^{\mathrm{e}}$ siècle et qu'elle était revendiquée autant par la nouvelle République de Weimar que par le tout jeune État polonais, on pensa en 1921 qu'il serait possible de résoudre le problème par un plébiscite invitant la population locale à exprimer son vœu de rattachement à l'Allemagne ou à la Pologne. Les affiches et tracts rédigés lors de la campagne électorale pour ce plébiscite révèlent qu'on plaidait indifféremment dans les deux langues pour le rattachement à la Pologne ou à l'Allemagne et déconstruisent l'idée herdérienne que la nationalité d'une population se définit d'abord par sa langue.

En 1921, à la suite de ce plébiscite et des trois insurrections dites silésiennes qui en avaient contesté le résultat, la Haute-Silésie fut partagée entre l'Allemagne et la Pologne: tandis que cette dernière en obtenait la partie la plus riche et la plus industrieuse, Gleiwitz devenait ville frontière à l'extrême est de la République de Weimar. Cette construction fut remise en question en 1945, lorsque les accords de Potsdam décidèrent de repousser la frontière germano-polonaise sur l'Oder et la Neiße, et de remettre ainsi à la Pologne l'intégralité de la Haute-Silésie allemande et avec elle Gleiwitz, appelée dorénavant Gliwice.

Bien qu'elle se trouve aujourd'hui à plus de $300 \mathrm{~km}$ de la frontière allemande, toute la région garde l'empreinte de son ancienne et longue appartenance à l'Allemagne. On pense en premier lieu aux prestigieuses traces architecturales qu'a laissées l'extrême dynamisme industriel de la fin du $\mathrm{XIX}^{\mathrm{e}}$ siècle. C'est aussi, comme le révèle le recensement de 2011, la région de Pologne dans laquelle on trouve le plus grand nombre des personnes déclarant appartenir à deux nationalités. On y affirme même l'existence d'une nationalité silésienne spécifique, qui ne serait ni allemande, ni polonaise ${ }^{(8)}$.

Pour comprendre la persistance de cette particularité régionale, il faut remonter à 1945 : après avoir fui massivement à l'approche de l'Armée rouge et avoir tenté de rentrer chez elle après l'arrêt des combats, une grande partie de la population allemande s'est fait expulser en deux temps, d'abord d'une façon que les Allemands disent « sauvage ", mais que les Polonais appellent plutôt " militaire », ensuite d'une façon légale et de plus en plus organisée en application des accords de Potsdam qui avaient attribué cette région à la Pologne.

Comme le nouveau pouvoir polonais ne pouvait pas expliquer à sa population que ces vastes régions lui étaient en réalité attribuées en dédommagement des territoires orientaux que l’Union soviétique avait confisqués dès 1939 à la Pologne, il rappelait qu'autrefois, au $\mathrm{X}^{\mathrm{e}}$ siècle, la frontière polonaise s'était trouvée sur l'Oder et affirmait que l'État polonais ne faisait maintenant que récupérer des terres historiquement polonaises, «terres recouvrées » (ziemie odzyskane) dont la Haute-Silésie était la partie la plus industrielle.

7 Voir Pascal Fagot, Mémoires et regards: le thème de la Pologne dans la prose littéraire allemande (19491990), Berne, Peter Lang, 2001.

8 http://stat.gov.pl/spisy-powszechne/nsp-2011/nsp-2011-wyniki/ludnosc-stan-i-struktura-demograficznospoleczna-nsp-2011,16,1.html. 
Après 1945, à la suite d'une guerre extrêmement brutale pour la population polonaise, le gouvernement polonais entreprit de faire de la Pologne l'État national qu'elle n'avait jamais été et décida de ne garder sur son territoire qu'une population répondant à sa définition de la polonité, c'est-à-dire d'abord polonophone. À cette fin, il fallait que les habitants allemands de Haute-Silésie qui n’avaient pas encore quitté la région soient « rapatriés » en Allemagne pour laisser place à des populations polonophones venues d'autres régions de Pologne, et notamment des territoires attribués à l'Union soviétique.

Pour réaliser ce programme, il semblait qu'il serait simple de répartir la population haut-silésienne en deux groupes, c'est-à-dire de séparer les Allemands des Polonais et d'obliger les premiers à quitter la Pologne pour permettre aux Polonais qui resteraient et à ceux qui arriveraient du centre ou de l'est du pays de participer à la construction d'une nouvelle Pologne véritablement polonaise.

On créa alors des commissions dont la fonction était de "vérifier » la nationalité des habitants de Haute-Silésie qui, pour diverses raisons, demandaient à ne pas être expulsés et qui, en cas de réponse positive de la part de la commission, s'engageaient à devenir de «vrais Polonais » et à se comporter comme tels.

Le principe paraissait simple, mais son application s'avéra très complexe, d'abord parce qu'il était souvent difficile de désigner la nationalité des personnes bilingues qui se soumettaient à cette entreprise de vérification. En outre, les membres des commissions de vérification n'avaient pas été formés à cette tâche, ils étaient souvent étrangers à cette région dont ils ne comprenaient pas le fonctionnement et se laissaient guider dans leur décision par toutes sortes de motivations qui empêchaient un jugement impartial; enfin, le principe était inapplicable, car, par-delà toute considération d'ordre national, il fallait retenir les personnes compétentes ou "spécialistes » qui puissent faire fonctionner les usines et les mines de la région et qui, souvent, se définissaient comme allemandes.

\section{Après 1945 : une révolution patrimoniale}

Après 1945, il fallait donc que vivent ensemble en Haute-Silésie des populations dont les histoires étaient extrêmement différentes. Tandis que les uns avaient été citoyens allemands et avaient combattu sous l'uniforme de la Wehrmacht, les autres, plus nombreux, avaient vécu entre les deux guerres dans la République de Pologne et revêtu l'uniforme de l'armée polonaise vaincue par les premiers; ils arrivaient du Gouvernement général ou bien encore des régions que la Pologne avait dû céder à l'Union soviétique; en remontant un peu dans le temps, on se souvenait même qu'avant 1918, ils avaient été citoyens russes, autrichiens ou allemands/prussiens.

Pour homogénéiser le nouveau groupe que devaient former toutes ces populations aux origines si diverses, il fallait leur constituer un patrimoine culturel commun qui représenterait leurs « valeurs » et leurs «traditions » et qu'elles transmettraient par la suite en héritage aux générations suivantes. Après 1945, les anciens citoyens allemands qui avaient choisi de rester, ou dont les compétences étaient si nécessaires à la bonne marche de l'économie qu'on leur avait refusé l'autorisation de quitter la Pologne, se virent donc contraints de rompre avec leur passé allemand et d'opérer une véritable « révolution patrimoniale» au cours de laquelle il leur fallut adopter le patrimoine que leur avait créé leur nouvel État. 
La rupture fut évidemment d'abord d'ordre linguistique. Il n'était dorénavant pas seulement interdit de parler allemand en tous lieux, c'est toute trace écrite de la langue allemande qui devait disparaître des espaces public et privé. Pour cela, les Silésiens, qui n'avaient jamais fréquenté d'école polonaise, durent apprendre et utiliser la langue de l'État dont ils étaient les nouveaux citoyens. Les anciens Allemands qu'ils étaient devaient oublier tout ce que l'Allemagne leur avait appris à l'école, dans l'entreprise, et dans l'armée: outre les vers de Goethe, de Schiller et d'Eichendorff ${ }^{(9)}$, il leur fallait rayer de leur mémoire qu'ils avaient autrefois fêté l'anniversaire de l'Empereur allemand, que leurs parents et grands-parents avaient en leur temps chanté Die Wacht am Rhein ${ }^{(10)}$ et fêté la victoire de Sedan, qu'ils avaient participé à deux guerres mondiales sous l'uniforme allemand, que, depuis le $\mathrm{XIX}^{\mathrm{e}}$ siècle, ils avaient pris part au spectaculaire développement économique et industriel de l'Allemagne et que, il y a seulement quelques années, ils avaient voté pour les partis Zentrum, SPD, KPD ou bien encore NSDAP.

Pour qu'ils puissent se constituer une nouvelle mémoire polonaise, on entreprit de détruire en Haute-Silésie devenue polonaise tout ce qui avait servi de lieu de mémoire allemand, non seulement les monuments élevés par les nazis, mais aussi ceux que le nationalisme avait construits au XIX ${ }^{\mathrm{e}}$ siècle pour commémorer Bismarck et la création du Reich ${ }^{(11)}$ et on les remplaça par de nouveaux monuments évoquant l'histoire polonaise. On instaura un nouveau calendrier de fêtes nationales, on offrit à la population haut-silésienne de nouveaux héros nationaux, et on lui raconta de nouveaux mythes fondateurs, parmi lesquels les insurrections silésiennes du début des années 1920 et la bataille du Annaberg ${ }^{(12)}$; on lui expliqua qu'elle avait vécu pendant des siècles sous l'oppression allemande, mais qu'elle retrouvait sa véritable histoire qui avait commencé au début du millénaire avec la dynastie des Piast et qui, après une trop longue interruption, pouvait enfin reprendre son cours normal.

Au grand discours national allemand, on substitua donc un grand discours national polonais qui présentait le rattachement de la Haute-Silésie à la Pologne comme l'heureux aboutissement de luttes ancestrales. De la même façon, on imposa aux Polonais venus de l'est de la Pologne d'oublier qu'ils étaient nés sur le territoire de ce qui était maintenant une République de l'Union soviétique, et qu'ils en avaient été expulsés; on leur expliqua qu'ils avaient été " rapatriés » en Pologne, et on exigea qu'ils adhérent à ce nouveau discours patrimonial justifiant leur présence dans cette ancienne région allemande dans laquelle ils devraient apprendre à vivre.

On ne créait donc pas seulement une nouvelle population haut-silésienne extraordinairement bigarrée par la diversité de ses origines géographiques et culturelles, on lui offrait un nouveau " patrimoine " préfabriqué, un ensemble de biens matériels et immatériels qu'elle devait s'approprier et qui contribuerait à établir sa nouvelle appartenance à une nation polonaise remodelée par les amples mouvements de population qui suivirent la Seconde Guerre mondiale.

9 Né en Haute-Silésie, le poète Joseph von Eichendorff est un des personnages emblématiques de la région.

10 S. Rosenbaum, Germania (note 6), p. 66.

11 Voir le monument aux morts de la guerre prusso-française de 1870-1871 dans le catalogue de l'exposition: S. Rosenbaum, Germania (note 6), p. 84-85.

12 Lieu de pèlerinage où, en mai 1921 dans le cadre des insurrections silésiennes, les partisans d'un rattachement de la Haute-Silésie à l'Allemagne affrontèrent les partisans d'un rattachement à la Pologne. 


\section{L'exposition Germania de 2012}

Ce récit fit autorité tant que le parti communiste qui en était l'auteur fut au pouvoir, c'est-à-dire jusqu'en 1990. Après la chute de l'Union soviétique et avec les premiers gouvernements non communistes en Pologne, les historiens polonais profitèrent de leur nouvelle liberté pour réécrire l'histoire de leur pays et en faire émerger des pans entiers qui en avaient été dissimulés. C'est ainsi qu'ils réexaminèrent l'histoire de la relation avec l'Union soviétique, qui fait l'objet principal des recherches de l'IPN (Instytut Pamięci Narodowej/Institut de la mémoire nationale $\left.{ }^{(13)}\right)$, mais aussi l'histoire de la relation avec les deux États allemands ${ }^{(14)}$. Ils s'intéressèrent tout particulièrement aux expulsions des Allemands hors de Pologne ${ }^{(15)}$ et à l'histoire des « terres recouvrées » ${ }^{(16)}$.

Ainsi réapparut peu à peu l'histoire allemande de la Haute-Silésie qu'on osait maintenant désigner comme une spécificité régionale. C'est dans ce contexte que se situe l'exposition Germania, organisée en 2012 par le musée de Gliwice, dont il sera maintenant question.

L'exposition portait le titre de Germania, la vie politique allemande en Haute-Silésie 1871-1945 ${ }^{(17)}$. Elle était accompagnée d'un ouvrage que nous appellerons catalogue, même s'il ne mérite pas vraiment ce nom car il ne reproduit qu'une petite partie des objets exposés; il est intéressant par le fait qu'il complète l'exposition par un exposé explicatif de l'auteur dans lequel ce dernier recourt non seulement à des travaux d'historiens universitaires ${ }^{(18)}$, mais aussi à ceux d'historiens régionaux et à des extraits de textes rédigés par d'anciens habitants de la région ${ }^{(19)}$.

L'exposition était organisée d'une manière chronologique en trois périodes réparties dans trois salles:

- De l'unification allemande jusqu'à la Grande Guerre (1871 à 1918)

- L'entre-deux-guerres jusqu'en 1939

- À l'ombre de la Seconde Guerre (1939-1945).

13 http://ipn.gov.pl/

14 Par exemple: Jerzy Kochanowski, Klaus Ziemer (dir.), Polska - Niemcy wschodnie 1945-1990. Wybór dokumentów (Pologne - Allemagne Orientale 1945-1990. Choix de documents), Varsovie, Neriton, t. 1, 2006; t. 3, 2008. Également: Krzysztof Ruchniewicz, Warszawa-Berlin-Bonn. Stosunki polityczne 1949-1958 (Varsovie-Berlin-Bonn. Relations politiques 1949-1958), Wrocław, Wydawnictwo Uniwersytetu Wrocławskiego, 2003.

15 Voir notamment les quatre tomes du recueil de documents historiques: Włodzimierz BorodziEj et Hans Lemberg (dir.), Niemcy w Polsce (Les Allemands en Pologne), Varsovie, Neriton, 2000. Version allemande: "Unsere Heimat ist uns ein fremdes Land geworden..." Die Deutschen östlich von Oder und Neiße 1945-1950. Dokumente aus polnischen Archiven, Marbourg, Verlag Herder Institut, 2000. Voir aussi: Maria Podlasek, Wypędzenie Niemców z terenów na wschód od Odry i Nysy Łyżyckiej. Relacje świadków (L'expulsion des Allemands hors des territoires situés à l'est de l'Oder et de la Neiße de Lusace. Récits de témoins), Varsovie, Wydawnictwo polsko-niemieckie, 1995. Également: Stanisław Janкоwiak, Wyjazdy Niemców z Polski w latach 1950-1959. Wybór dokumentów (Le départ des Allemands de Pologne dans les années 1950-1959. Choix de documents), Poznań, Wydawnictwo Poznańskie, 2010.

16 Par exemple : Robert Traba, Wschodniopruskość (La prussianité orientale), Poznań/Varsovie, Borussia, 2005.

17 Germania. Niemiecka polityczność na Górnym Śląsku 1871-1945.

18 Citons notamment Hans-Ulrich Wehler, Thomas Nipperdey et Heinrich August Winkler.

19 Il s'agit de récits autobiographiques, d'extraits de roman, de journaux locaux, etc. 
Plus détaillé, le catalogue est composé d'une douzaine de chapitres dont certains titres reprennent des formules qui ont marqué l'histoire allemande telles que Kulturkampf, Par le fer et le sang (Żelazem i krwia/Blut und Eisen) ou La trahison de Versailles (Wersalska zdrada/Versailler Verrat).

En structurant son travail à partir du calendrier de l'histoire allemande et en écartant toute référence à l'histoire polonaise, l'auteur du catalogue annonce une double rupture avec les discours dominants sur la Haute-Silésie; il signifie en effet qu'il ne reprendra ni le discours polonais sur les «terres recouvrées» ni celui des associations d'expulsés allemands qui ont pour premier objectif d'en affirmer la "germanité », mais qui n'en passent pas moins sous silence de larges pans de l'histoire allemande de la région ${ }^{(20)}$.

Tout d'abord, l'exposition Germania souligne que, de 1866 à 1945, de la guerre contre l'Autriche alors toute proche jusqu'à la capitulation sans conditions du III ${ }^{\mathrm{e}}$ Reich en passant par les guerres de 1871, de 1914-1918 et de 1939-1945, la Haute-Silésie et la ville de Gliwice ont activement participé à l'histoire militaire prussienne puis allemande. Elle montre également que la région a partagé l'intégralité de l'histoire politique allemande qui fit passer l'Allemagne du Deuxième Empire à la tentative de révolution de 1918, de la République de Weimar à la dictature du III ${ }^{\mathrm{e}}$ Reich, de la guerre à la capitulation et à l'expulsion des Allemands.

Elle insiste enfin sur le fait que la Haute-Silésie a joué un rôle essentiel dans l'histoire économique de l'Allemagne: elle n'a pas seulement apporté une contribution décisive à son formidable essor industriel de la fin du XIX ${ }^{\mathrm{e}}$ siècle et aux bouleversements sociaux que ce dernier engendra, elle a également souffert de la violence de la crise économique des années 1920 qui a plongé la plus grande partie de sa population dans une misère profonde ${ }^{(21)}$; ses abondantes ressources ont ensuite été exploitées par les maîtres d'œuvre du national-socialisme qui y ont instauré une économie de guerre malmenant brutalement la main-d'œuvre étrangère employée dans les mines et usines locales et parquée dans d'innombrables camps de travail.

En choisissant cette chronologie, les organisateurs de l'exposition composent un récit révélant la complexité des conflits sociaux, politiques et économiques qui ont agité la Haute-Silésie entre 1871 et 1945 et suggèrent que, par-delà les stéréotypes, la vie politique de la région n'était pas réductible au conflit germano-polonais que le pouvoir communiste polonais et les associations de réfugiés avaient, ensemble et l'un contre l'autre, toujours mis en avant.

La deuxième conclusion à laquelle nous mènent cette exposition et l'ouvrage qui l’accompagne tient à la qualité des objets présentés qui, en lui restituant sa matérialité, font revivre cette histoire à la fois niée et oubliée. Les Polonais de 1945 avaient bien

20 C’est ainsi que, dans sa présentation de la Haute-Silésie, le site de la Fédération des expulsés (Bund der Vertriebenen) ne mentionne la Seconde Guerre mondiale que pour chiffrer le nombre des victimes haut-silésiennes: http://www.z-g-v.de/zgv/fakten-und-hintergruende/die-geschichte-der-deutschenvertriebenen/\#c266: «Von den über 1,5 Millionen Einwohnern der Provinz Oberschlesien (in ihren Vorkriegsgrenzen ohne das im Oktober 1939 angegliederte Ost-Oberschlesien) kamen $85.000 \mathrm{im}$ Krieg um, 570.000 wurden bis 1950 vertrieben, zu über 90 Prozent nach Westdeutschland. »

21 S. Rosenbaum, Germania (note 6), p. 190-191. 
compris la force des objets et savaient que le passé allemand ne passerait pas aussi longtemps que des objets pourraient en témoigner, c'est pourquoi, et bien que cela soit irréalisable, ils avaient tenu à ce que disparaisse toute trace matérielle du passé allemand de la région ${ }^{(22)}$. En 2012, le musée de Gliwice présentait pour la première fois ces objets longtemps interdits dans l'espace public polonais. Il exposait un grand nombre de photographies privées, mais aussi des documents administratifs, des uniformes des différentes armées allemandes qu'avaient portées les habitants de Gliwice, de la correspondance privée, autant de pièces qui témoignaient de l'implication de la population locale dans le passé allemand de la région. Dans le sens propre du mot témoin qui raconte sa présence à l'événement, ces objets et ces photos de Haut-silésiens souvent anonymes apportaient dans le présent polonais les preuves matérielles de l'existence d'un ancien présent allemand.

L'exposition restituait la langue allemande dans la fonction qui avait été la sienne dans cette région jusqu'en 1945: langue de l'administration, de l'école, de la bourgeoisie, de l'aristocratie, de l'armée, de la politique, de la culture, de la vie associative. Les photos et documents représentaient les Haut-Silésiens comme loyaux sujets de l'Empereur et bons patriotes allemands, ils racontaient la quiète assurance des élites bourgeoise et aristocratique locales et démontraient leur engagement dans la vie politique et économique de la Prusse et du Reich. Ils les faisaient apparaître comme acteurs importants de la vie mondaine berlinoise et comme le principal vecteur du nationalisme et de la culture allemande.

Loin de toute idéalisation d'une Heimat hors du temps, loin donc du Heimatroman inspirant tant de récits des victimes de la fuite et de l'expulsion, les nombreux objets exposés ne suggéraient pas seulement la diversité conflictuelle de la société, ils révélaient que la population de Haute-Silésie avait entendu et repris à son compte tous les discours qui avaient eu cours en Allemagne, qu'il s'agisse de celui du Kulturkampf ou de l'antisémitisme du XIX ${ }^{e}$ siècle, de celui de la révolution de 1918 ou du nationalsocialisme triomphant d'après 1933. Ils établissaient que cette population avait tout ensemble été victime et actrice du système de répression national-socialiste, qu'elle avait accueilli sur son territoire et qu'elle avait aidé à faire fonctionner d'innombrables camps de travail pour soldats soviétiques et autres victimes du nazisme ${ }^{(23)}$ jusqu'à ce qu'elle subisse de plein fouet les bombardements et la catastrophe finale de 1945 .

Certes, les organisateurs présentaient certaines spécificités régionales tenant au fait qu'une partie de la population était bilingue et que le mouvement nationaliste polonais avait réussi à prendre racine en Haute-Silésie. Mais là n'était pas son propos principal qui, à l'encontre du discours polonais longtemps dominant, était en réalité de présenter la Haute-Silésie comme une région qui avait été entièrement intégrée dans la sphère culturelle et politique de l'Allemagne, tournée qu'elle était vers Berlin et le Reich et agitée par tous les conflits qu'avait connus l'Allemagne.

22 Cette thématique revient très fréquemment dans les documents présentés dans Niemcy $w$ Polsce (note 15).

23 Les pages 226 et 227 du catalogue reproduisent les photos de soldats SS employés dans la filiale de Gleiwitz du camp d'Auschwitz. 
L'entreprise était certainement critiquable pour le choix qu'elle avait fait de présenter la vie politique haut-silésienne à partir de documents servant l'auto-représentation d'une société (défilés militaires, photos de groupes ou manifestations solennelles disant somme toute assez peu sur la vie quotidienne et la psychologie de la population) et, parce que, comme l'annonçait son titre, elle se focalisait sur la vie politique allemande et devait nécessairement négliger la vie politique polonaise, mais elle rendait à la Haute-Silésie l'histoire allemande que la Pologne communiste lui avait confisquée et que, jusqu'alors, on rechignait encore dans ce pays à vouloir reconnaître.

\section{Conclusion : peut-on parler ici de «patrimonialisation du passé allemand »?}

Pour répondre à cette question, revenons à la définition du patrimoine que nous fournit le Conseil de l'Europe: le patrimoine culturel serait un ensemble de ressources héritées du passé qu’une communauté considère comme reflet de ses valeurs, de ses croyances et de ses traditions et qu'elle tient pour dignes d'être transmises à de nouvelles générations. La visite de l'exposition et la lecture du texte l'accompagnant mènent à la conclusion qu'elle ne répond pas à deux des critères définissant le patrimoine et qu'on ne peut donc parler ici d'une patrimonialisation du passé allemand.

$\grave{A}$ aucun moment, l'auteur du texte et les organisateurs de l'exposition ne prennent la parole au nom d'une communauté patrimoniale nationale (allemande ou polonaise), ou même régionale, même si la place réservée dans le catalogue à certains personnages peut donner à penser que l'auteur du texte n'est pas insensible aux arguments régionalistes ${ }^{(24)}$. L'exposition et le texte l'accompagnant sont destinés au grand public. Le narrateur prend son lecteur par la main, il l'inclut dans un "nous » qui examine une histoire passée, une société devenue étrangère qu'il s'agit d'expliquer. Le texte n'a ni la structure, ni la rigueur d'un véritable travail d'historien, c'est une promenade à travers des documents et des mémoires qui tentent de faire revivre une époque. Le « nous » qu'il met en place a pour objectif de faciliter la lecture du texte; il s'agit d'un " nous, qui vivons aujourd'hui », qui ne se réfère ni aux Allemands, que le narrateur appelle Allemands, ni aux Polonais, qu'il appelle Polonais, ni même aux Haut-Silésiens, qu'il appelle Haut-Silésiens. On peut d'ailleurs se demander quelle aurait pu être la nationalité de ce " nous » auquel les auteurs de l'exposition auraient pu se référer. Pouvait-ce être le «nous » des Haut-silésiens dont la famille a une histoire allemande, au risque d'exclure ainsi les nombreux habitants de la région ne partageant pas cette particularité? Pouvait-il renvoyer à une communauté polonaise qui aurait exclu les personnes dont une partie de l'histoire était allemande et qui aurait avec elles privé la Haute-Silésie d'une part essentielle de son passé?

Les organisateurs de l'exposition n’ont pas sélectionné parmi l'histoire allemande de la région des aspects spécifiques qu'ils auraient voulu mettre en valeur et promouvoir

24 Nous pensons ici aux nombreuses pages que l'auteur consacre à Anton Korczok, un prêtre catholique haut-silésien qu'il désigne comme martyr. Peu soucieux de distinguer entre catholiques allemands et polonais et venant en aide aux uns comme aux autres, il est décédé au camp de Dachau, victime des nazis allemands; pourtant, après la guerre, les communistes polonais ont fait effacer son nom sur sa tombe pour la raison que l'inscription était en langue allemande (S. Rosenbaum, Germania [note 6], p. 196-207). 
parce qu'ils les considéraient comme dignes d'être transmis en héritage. C'est, à la mesure de l'espace dont ils disposaient, l'intégralité de l'histoire allemande de la région qu'ils relatent, sans en omettre les aspects les plus sombres ou les plus brutaux que l'on a d'ordinaire tendance à exclure des patrimoines parce qu'on ne veut pas s'y reconnaître. En ce sens, il ne nous semble pas qu'on puisse ici parler d'une patrimonialisation de l'histoire allemande par les Polonais, contrairement à ce qu'a proposé par exemple une exposition réalisée en 2010 à Varsovie par la Stiftung für deutschpolnische Zusammenarbeit ${ }^{(25)}$ et la Maison de rencontres avec l'histoire ${ }^{(26)}$ sur l'histoire de la population allemande de Varsovie ${ }^{(27)}$ dont l'objectif évident était de valoriser sa contribution au développement de la ville et de l'intégrer ainsi dans le patrimoine national.

Plutôt que de patrimonialisation, nous parlerons ici d'une double dépatrimonialisation de la Haute-Silésie par un récit qui est autant en rupture avec l'ancien récit patrimonialisant du pouvoir polonais qu'avec le récit patrimonialisant d'une grande partie des expulsés allemands. L'exposition est dépatrimonialisante par le fait qu'elle adopte la position de l'historien et s'efforce de la faire partager par son public. Sans vouloir opérer de sélection, sans valoriser tel ou tel événement, elle rend à la HauteSilésie la complexité de son histoire allemande, dont les uns et les autres l'avaient privée. Elle ne commémore pas et ne cherche pas à transmettre des valeurs, elle s'efforce de faire comprendre une situation historique et, pour cela, dénationalise le discours sur la région. Elle semble vouloir montrer que, plus que d'un patrimoine culturel, c'est d'une véritable histoire explicative que nous avons besoin et que, s'il faut transmettre un patrimoine aux générations à venir, c'est l'histoire tout entière qui doit entrer dans ce patrimoine.

\section{Résumé}

Après avoir défini la patrimonialisation du passé comme un récit fondé sur la sélection par une communauté d'un ensemble de biens matériels et immatériels considérés comme dignes d'être transmis aux générations à venir, l'auteur rappelle que le gouvernement polonais avait tenté après 1945 de proposer à la population allemande restée en Haute-Silésie devenue polonaise un nouveau récit national dont l'objectif était, par la patrimonialisation d'un très ancien passé polonais de la région, de l'intégrer dans le nouveau corps national. En s'appuyant sur une exposition réalisée en 2012 à Gliwice (Pologne), il expose dans un dernier temps une façon alternative de présenter le passé régional déconstruisant les différentes patrimonialisations des passés nationaux. Cette "dépatrimonialisation" se ferait au profit d'une approche ne fondant pas un nouveau discours national ou régional qui sélectionnerait dans le matériau historique les seuls

25 http://sdpz.org/

26 Dom Spotkań z Historia, ul. Karowa 20, 00-324 Warszawa.

27 Tomasz Markiewicz, Tadeusz Władysław Świątek, Krzysztof Wittels, Polacy z wyboru. Rodziny pochodzenia niemieckiego $w$ Warszawie $w$ XIX $i \mathrm{xx}$ wieku (Polonais par choix. Les familles d'origine allemande dans la Varsovie du XIX ${ }^{e}$ et du $\mathrm{XX}^{\mathrm{e}}$ siècle), Varsovie, Wydawnictwo Dom Spotkań z Historią, 2012. 
éléments considérés comme dignes d'être retenus et transmis aux générations suivantes, mais dont l'objectif serait, plutôt que d'en commémorer certains épisodes choisis, de "dénationaliser " le regard historique pour considérer l'histoire tout entière et ainsi mieux la faire comprendre.

\title{
Zusammenfassung
}

Patrimonialisierung der Vergangenheit definiert der Autor als eine auf der Auswahl bestimmter materieller und immaterieller Güter basierende Erzählung, die bei der Gestaltung einer gemeinsamen Identität helfen soll. Er erklärt, wie es nach 1945 die polnische Regierung unternommen hatte, der in Polen verbleibenden deutschen Bevölkerung eine neue patrimonialisierende Erzählung der polnischen Vergangenheit der Region aufzuzwingen und von ihr verlangt hatte, dass sie die ehemalige deutsche patrimonialisierende Erzählung vergesse.

Anhand einer Ausstellung in Gliwice/Gleiwitz (Polen, Oberschlesien) legt dann der Autor dar, wie Vergangenheit alternativ dargestellt werden kann, indem sowohl auf die deutsche als auch auf die polnische Patrimonialisierung der regionalen Geschichte verzichtet wird zugunsten einer historisierenden Herangehensweise, die keine patrimonialisierende Auswahl im geschichtlichen Material triff, und auch keinen besonderen nationalen oder gar regionalen Erinnerungsort auszumachen trachtet, sondern den historischen Blick entnationalisiert und auf diese Weise die ganze Geschichte darlegen, erklären und somit entpatrimonialisieren möchte.

\begin{abstract}
After having defined the patrimonialisation of the past as a narrative based on the selection by a community of a set of tangible and immaterial properties considered as worthy being transmitted to the next generations, the author recalls that the Polish government had tried after 1945 to impose to the German population which remained in High-Silesia a new national narrative whose objective it was, by the patrimonialisation of an old Polish past of the region to integrate it in the new national body.

On the basis of an exposition in 2012 in Gliwice (Poland, Upper Silesia), the author exposes an alternative way to present the regional past keeping aloof from the various patrimonialisations of the national pasts. This "depatrimonialisation" would be done with the profit of an approach not basing on a new national or regional narrative which would select in historical material the only elements considered as worthy being retained and transmitted to the following generations; his objective would be, rather than to commemorate certain episodes chosen of them, "to denationalize" the historical narrative and to render it comprehensible by considering the very whole history.
\end{abstract}

